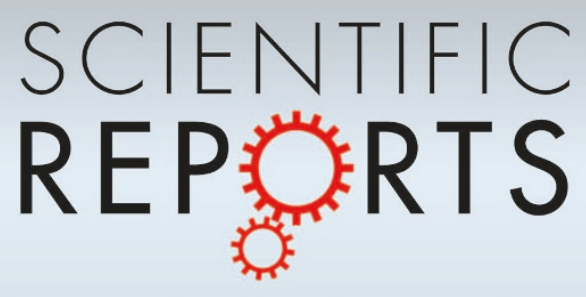

OPEN

SUBJECT AREAS:

SEMICONDUCTORS

ELECTRONIC DEVICES

CONDENSED-MATTER PHYSICS

Received

19 August 2014

Accepted

9 October 2014

Published

30 October 2014

Correspondence and requests for materials should be addressed to

K.T. (tanimura@

sanken.osaka-u.ac.jp)

\section{State-resolved ultrafast dynamics of impact ionization in $\operatorname{lnSb}$}

\author{
H. Tanimura, J. Kanasaki \& K. Tanimura
}

The Institute of Scientific and Industrial Research, Osaka University, 8-1, Mihogaoka, Ibaraki, Osaka 567-0047, Japan.

Impact ionization (IMP) is a fundamental process in semiconductors, which results in carrier multiplication through the decay of a hot electron into a low-energy state while generating an electron-hole pair. IMP is essentially a state selective process, which is triggered by electron-electron interaction involving four electronic states specified precisely by energy and momentum conservations. However, important state-selective features remain undetermined due to methodological limitations in identifying the energy and momentum of the states involved, at sufficient temporal resolution, to reveal the fundamental dynamics. Here we report state-resolved ultrafast hot electron dynamics of IMP in InSb, a semiconductor with the lowest band-gap energy. The ultrafast decay of state-resolved hot-electron populations and the corresponding population increase at the conduction band minimum are directly captured, and the rate of IMP is unambiguously determined. Our analysis, based on the direct knowledge of state-resolved hot electrons, provides far deeper insight into the physics of ultrafast electron correlation in semiconductors.

MP is an important process induced by Coulomb interactions among electrons in semiconductors ${ }^{1,2}$. It critically affects carrier transport under high-electric field conditions ${ }^{3}$, and is of currently studied as a means to improve photovoltaic-device efficiency ${ }^{4-6}$. Extensive studies have been carried out on IMP in semiconductors using a variety of experimental and theoretical techniques ${ }^{7-16}$. Traditional experimental studies primarily measure the electric-field dependence of carrier multiplication ${ }^{7}$. Apart from the difficulty in correlating the electric field with the excess energy $\mathrm{E}_{\mathrm{ex}}$ of carriers, information concerning state-selective characteristics is completely lost by the energy-integrating nature of this method. Additional spectroscopic studies ${ }^{8-12}$, which could reduce these drawbacks, neither specify the pertinent energy and momentum states nor determine the absolute rate directly. The rate of IMP has been analytically formulated for a simple case $e^{1,17}$, but there have been no experimental results for comparison with those predicted by this formula, leaving our fundamental understanding of the IMP process ambiguous and incomplete. Therefore, it is critical to measure quantum-mechanical dynamics of IMP on a state-resolved basis.

Here we directly capture state-resolved dynamics of IMP in InSb, using angle- and time-resolved photoemission spectroscopy. InSb crystals with (110) surfaces were excited by fs-laser pulses, and photoinjected hot electrons were detected using time-delayed $4.71-\mathrm{eV}$ probe pulses. By detecting photoelectrons as a function of energy $\varepsilon$ and emission angle $\theta$, from the surface normal, we directly determine energy- and momentum-resolved distributions of hot electrons at fs-temporal resolution.

\section{Results}

Figure 1 (a) shows a photoemission map, at a time delay $(\Delta \mathrm{t})$ of $50 \mathrm{fs}$, as a function of $\varepsilon$ and $\theta$. The white broken and solid curves are the energy dispersions of the conduction band along the $\Gamma$-X and $\Gamma$-L lines ${ }^{18}$. The pump pulse injects electrons at energies of $1.10 \mathrm{eV}, 0.76 \mathrm{eV}$, and $0.40 \mathrm{eV}$ above the conduction-band minimum (CBM) from the heavy-hole $(\mathrm{HH})$, light-hole $(\mathrm{LH})$, and split-off $(\mathrm{SO})$ valence band, respectively. Importantly, the transition from the $\mathrm{HH}$ band around $\Lambda$ injects hot electrons predominantly into the $\mathrm{L}$ valley. The angle- and energyintegrated intensity of the peaks from the $\mathrm{HH}$ band shares more than $80 \%$ of total photoemission intensity generated by the pump pulse which creates carriers at a density of $4.2 \times 10^{17} \mathrm{~cm}^{-3}$.

Figures 1 (b) and (c) display angle-integrated intensities of photoemissions from hot electrons and electrons near the CBM as a function of pump-probe delay, $\Delta \mathrm{t}$. Electrons injected from the SO valence band decay within $200 \mathrm{fs}$ after excitation, while those from LH band decay more rapidly within $100 \mathrm{fs}$. However, electrons injected into $\Gamma$ and $\mathrm{L}$ valleys from the $\mathrm{HH}$ band exhibit relatively slow decay, surviving until $\sim 800 \mathrm{fs}$. Interestingly, a new peak at $\mathrm{E}_{\mathrm{ex}}=0.63 \mathrm{eV}$ begins to appear at around $\Delta \mathrm{t}=400 \mathrm{fs}$. The most striking feature is that the population near the CBM increases dramatically at $\Delta \mathrm{t}<300 \mathrm{fs}$. As seen in Fig. 1 (b), the electrons injected from the HH band 


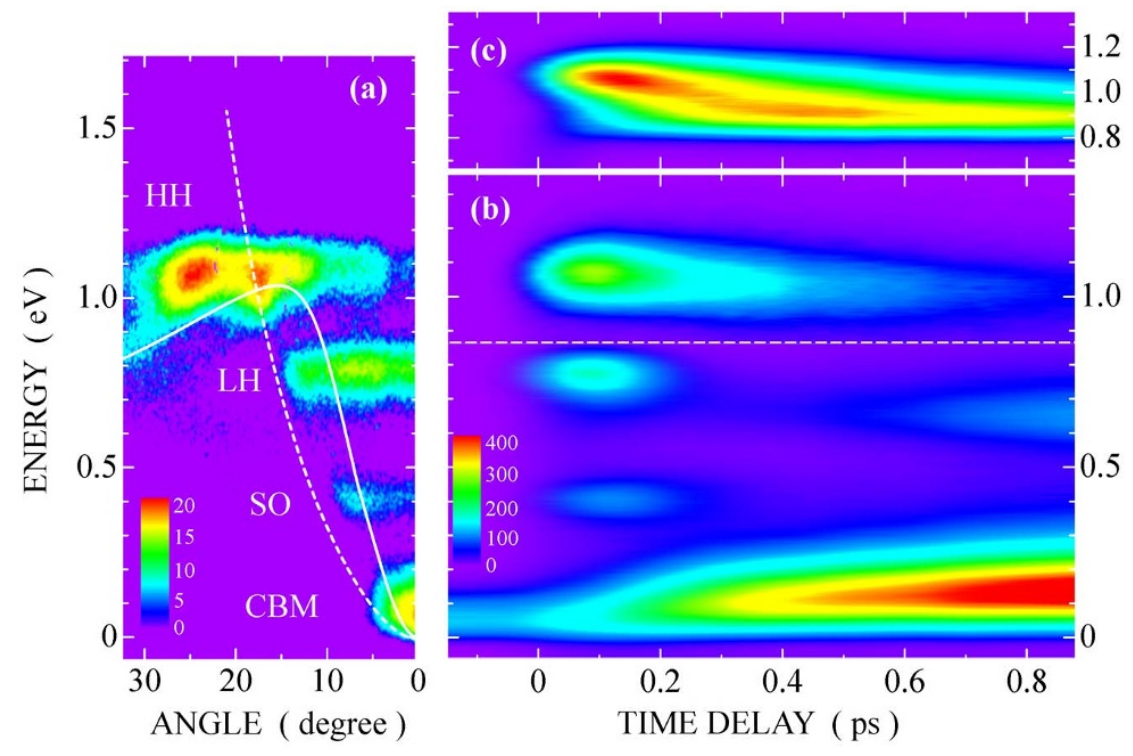

Figure $1 \mid$ Photoemission maps for the nascent electron distributions injected by 1.57-eV excitation and their subsequent dynamics in InSb.

(a) Photoemission image obtained using $1.57 \mathrm{eV}$ excitation pulses and 4.71-eV probe pulses delayed by 50 fs. Excitation and probe pulses are p-polarized. The color scale indicates the photoemission intensity as a function of electron energy and emission angle. The logarithmic intensities are plotted to clearly display regions of weak electron emission. The broken and solid white curves indicate the dispersions of the conduction band along $\Gamma$-X and $\Gamma$ - $\mathrm{L}$ directions as a function of emission angle (after Ref. 18). (b) Photoemission image of hot electrons injected into the $\Gamma$ valley by $1.57-\mathrm{eV}$ light pulses and dynamics of electrons populated near the CBM. (c) Photoemission image and dynamics of hot electrons injected into the L valley by 1.57 -eV light pulses. In (b) and (c), the angle-integrated photoemission intensities are plotted as a function of energy and time delay. The color scale indicates the angleintegrated intensity with a linear scale.

into the $\Gamma$ valley relax towards low energy states at a rate of $0.2 \mathrm{eV} / \mathrm{ps}$, which is too slow to reach the CBM within $300 \mathrm{fs}$. Also, low-lying hot electrons from the SO and $\mathrm{LH}$ bands do not show any low-energy shift during their respective decays. Therefore, the ultrafast increase in the electron population near the CBM results from fast IMP by hot electrons.

Figure 2(a) displays population dynamics near the CBM. At $293 \mathrm{~K}$, the population increases stepwise within $\Delta \mathrm{t}<300 \mathrm{fs}$, and reaches a maximum at around $\Delta \mathrm{t}=6 \mathrm{ps}$. The initial finite intensity at $\Delta \mathrm{t}<0$ comes from electrons thermally populated at the CBM at $293 \mathrm{~K}$ due to the small band-gap energy. The electron density at $\Delta \mathrm{t}<$ 0 as determined using spectral shape analysis is $3.0 \times 10^{16} \mathrm{~cm}^{-3}$ (see supplementary information). Based on this value, we determine that the population maximum corresponds to an electron density of $1.7 \times$ $10^{17} \mathrm{~cm}^{-3}$ at $\Delta \mathrm{t}=6 \mathrm{ps}$.

We can distinguish two distinctive processes in the growth of the population near the CBM: the fast process, at $\Delta \mathrm{t}<300 \mathrm{fs}$, and the slow process which follows it. During the slow process, the hotelectron population decreases in the $\mathrm{L}$ valley, and a new photoemission band at $\mathrm{E}_{\mathrm{ex}}=0.63 \mathrm{eV}$ develops. Temporal changes in the intensities of hot electrons in the $\mathrm{L}$ valley, angle-integrated for $\theta>$ $20^{\circ}$, and those populated at $\mathrm{E}_{\mathrm{ex}}=0.63 \mathrm{eV}$ in the $\Gamma$ valley are shown by blue and red curves in Fig. 2(b). The first feature represents the energy relaxation towards the $\mathrm{L}$ valley $\left(\mathrm{L}_{1}\right)$ minimum. We note that photoemissions beyond $\theta=35^{\circ}$ cannot be detected in our detection window, resulting in the observed intensity loss. A recent GW band calculation predicts $\mathrm{L}_{1}$ at $0.61 \mathrm{eV}$ above the $\mathrm{CBM}^{18}$, almost the same energy as $\mathrm{E}_{\mathrm{ex}}$ where the new band appears. Therefore, we conclude that the new band's appearance is due to the electrons scattered from $\mathrm{L}_{1}$ to the $\Gamma$ valley at the position, $\Gamma\left(\mathrm{L}_{1}\right)$. In fact, the rate equation model successfully correlates the relaxation in the $\mathrm{L}$ valley, the accumulation at $\mathrm{L}_{1}$, and the $\mathrm{L}-\mathrm{to}-\Gamma$ intervalley scattering, and satisfactorily describes the population dynamics at $\Gamma\left(\mathrm{L}_{1}\right)$ (see solid curves in Fig. 2(b)). The broken curve shows the predicted changes in the population at the minimum of the $\mathrm{L}$ valley.
Importantly, the L-to- $\Gamma$ intervalley scattering and the increase in population near the CBM takes place in parallel as shown in Fig. 2(c); both peaks near the CBM and electrons at $\Gamma\left(\mathrm{L}_{1}\right)$ are increasing concurrently. These results reveal that the hot electrons at the $\mathrm{L}_{1}$ induce both IMP and intervalley scattering competitively. In this IMP process, however, the momentum conservation among electronic states can no longer be satisfied, as no valence states exist on the line connecting $\mathrm{L}_{1}$ and the CBM, as shown by red broken line in Fig. 2 (d). Therefore, IMP by hot electrons at $\mathrm{L}_{1}$ is phonon-assisted ${ }^{2}$. The red curve in Fig. 2 (a) shows electron population dynamics near the CBM measured at $90 \mathrm{~K}$ under the same excitation condition as that measured at $293 \mathrm{~K}$. The enhancement in the slow process is dramatically suppressed at $90 \mathrm{~K}$ relative to that at $293 \mathrm{~K}$, supporting our conclusion that the IMP by hot electrons at the $\mathrm{L}_{1}$ is phonon-assisted.

In contrast to the slow process, the fast process is temperature insensitive. A stepwise increase in the population near the CBM is clearly detected at $90 \mathrm{~K}$ with almost the same enhancement as observed at $293 \mathrm{~K}$. Considering this result along with the ultrafast temporal response, we conclude that the fast process is due to the direct IMP by hot electrons in the $\Gamma$ valley. In fact, electrons injected from the SO and LH bands decay within this temporal domain as may be discerned from Fig. 1 (b), with decay times of $\tau_{\mathrm{d}}=150$ and $50 \mathrm{fs}$, respectively. In the direct IMP of hot electrons in the $\Gamma$ valley, interaction with the $\mathrm{LH}$ valence band is crucial $^{1,19,20}$. As seen in Fig. 2(d), hot electrons in the $\Gamma$ valley are under ideal conditions to satisfy required energy and momentum conservations because of the approximately linear dispersions of the conduction band and the $\mathrm{LH}$ valence band.

Since the electrons from $\mathrm{LH}$ band are injected above the energy of $\mathrm{L}_{1}$, they can be scattered into the $\mathrm{L}$ valley. To determine the effects of this intervalley scattering on $\tau_{\mathrm{d}}$, we excited InSb with light pulses at photon energies ranging from 1.2 to $1.57 \mathrm{eV}$, and determined the $\tau_{\mathrm{d}}$ 's of wave packets formed by photoinjection. Typical results are shown in Fig. 3(a). The electrons with $\mathrm{E}_{\mathrm{ex}}=0.53 \mathrm{eV}$ show $\tau_{\mathrm{d}}=150 \mathrm{fs}$, while those with $\mathrm{E}_{\mathrm{ex}}>0.65 \mathrm{eV}$ show shorter $\tau_{\mathrm{d}}$; the decay time of electrons 


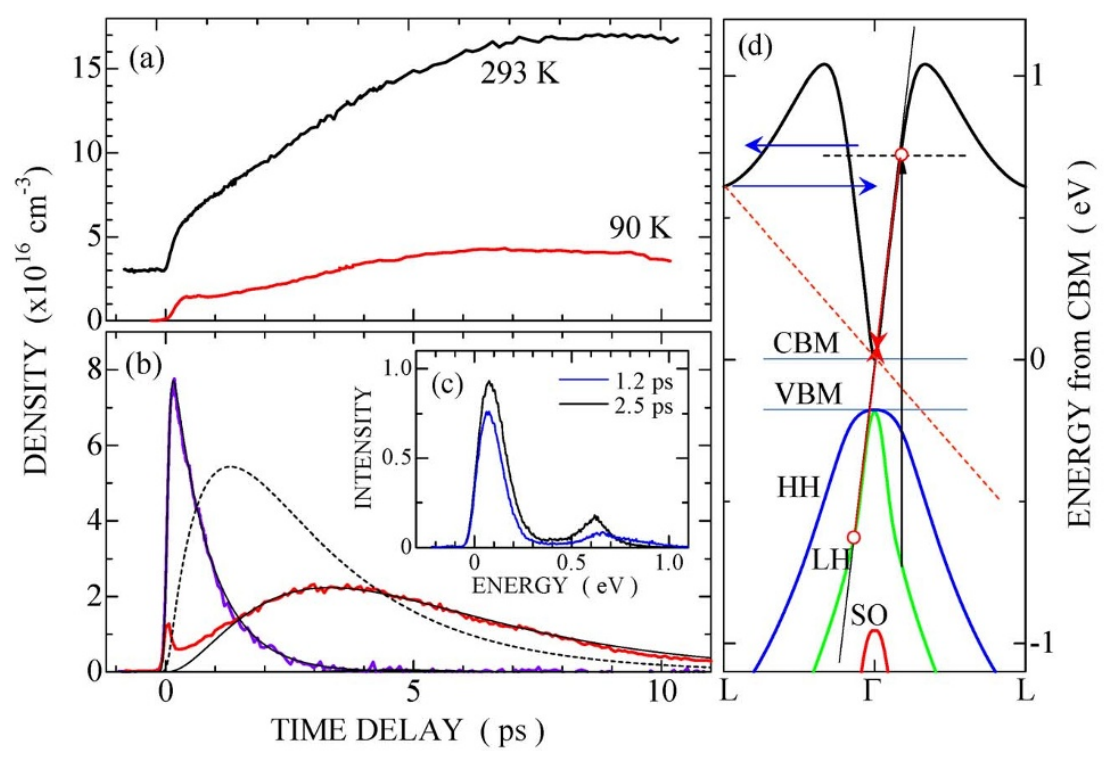

Figure $2 \mid$ Temporal changes in the population near the CBM and two processes of impact ionization. (a) Temporal changes in the electron population near the CBM measured at $90 \mathrm{~K}$ (red line) and $293 \mathrm{~K}$ (black line). Angle and energy integrated photoemission intensities (from $-5^{\circ}$ to $5^{\circ}$ and 0.1 to $0.35 \mathrm{eV}$ ) are plotted as a function of $\Delta \mathrm{t}$. The magnitude of the integrated intensity was converted to the electron density based on spectral-shape analysis (see the text). (b) Temporal changes in the intensities of hot electrons in the $\mathrm{L}$ valley (blue line) and those populated at $\Gamma\left(\mathrm{L}_{1}\right)($ red line). The same scaling factor to convert the integrated photoemission intensity into the electron density in (a) was assumed for these hot electrons. The thin solid curves are the results of the rate-equation analysis, showing temporal populations of relaxing electrons in the $\mathrm{L}$ valley and at $\Gamma\left(\mathrm{L}_{1}\right)$. The broken curve is the result obtained by the analysis, predicting temporal change in the population at the $\mathrm{L}_{1}$. (c) Angle integrated photoemission spectra $\left(-5^{\circ}\right.$ to $\left.5^{\circ}\right)$ measured at $\Delta t=$ 1.2 and 2.5 ps. The two peaks at 0.07 and $0.63 \mathrm{eV}$ represent populations near the $\mathrm{CBM}$ and at the $\Gamma\left(\mathrm{L}_{1}\right)$, respectively. (d) Dispersions along $\Gamma$-L direction for valence and conduction bands (after Ref. 18). The black arrow shows the optical transition from LH to the conduction band, and pathways of intervalley scattering from $\Gamma$-to-L and L-to- $\Gamma$ are shown by blue arrows. A possible direct impact ionization process is shown by red arrows. The red broken line connecting the $\mathrm{L}_{1}$ and the CBM represents the limit of the direct impact ionization process.

with $\mathrm{E}_{\mathrm{ex}}=0.79 \mathrm{eV}$ is as short as $50 \mathrm{fs}$. The relation between the transition rate, given by the inverse of $\tau_{\mathrm{d}}$, and $\mathrm{E}_{\mathrm{ex}}$ is plotted in Fig. 3 (b). It is clear that there is a critical energy of $\mathrm{E}_{\mathrm{ex}}=0.63 \mathrm{eV}$, exactly coincides with the energy of $L_{1}$, above which $\tau_{d}$ becomes dramatically shorter. Therefore, hot electrons with $\mathrm{E}_{\mathrm{ex}}>0.65 \mathrm{eV}$ undergo efficient scattering to the $\mathrm{L}$ valley, while those with $\mathrm{E}_{\mathrm{ex}}<$ $0.60 \mathrm{eV}$ do not.

\section{Discussion}

The results described above show that the hot electrons with $\mathrm{E}_{\mathrm{ex}} \approx$ $0.5 \mathrm{eV}$ decay with $\tau_{\mathrm{d}}=150$ fs. Radiative and phonon-assisted nonradiative recombination occurs at much slower rates ${ }^{21}$. The possible transient Auger recombination of the hot electrons, induced by photoinjected holes in the LH band, may be ignored as the excitation density of the LH band is at most $1.54 \times 10^{17} \mathrm{~cm}^{-3}$, which is much smaller than the density of states $\left(>1.0 \times 10^{18} \mathrm{~cm}^{-3}\right)$ of the LH band in the energy range where holes are injected. In fact, the decay times of hot electrons with $\mathrm{E}_{\mathrm{ex}}<0.6 \mathrm{eV}$ did not depend on the excitation density between $2.2 \times 10^{17}$ and $1.3 \times 10^{18} \mathrm{~cm}^{-3}$. Therefore, we conclude that the decay of these hot electrons is due to direct IMP. Furthermore, as shown in Fig. 3(b), we can determine directly the rate of direct IMP of electrons with $\mathrm{E}_{\mathrm{ex}}=0.5 \mathrm{eV}$ in InSb to be $1 / \tau_{\mathrm{d}}=$ $6.7 \times 10^{12} \mathrm{~s}^{-1}$.

The theory of IMP provides the following formula for the rate of IMP for a parabolic system:

$$
W_{I M P}=W_{0}\left(\frac{\varepsilon_{0}}{\varepsilon_{\infty}}\right)^{2} \frac{m_{c}^{*}}{m_{0}} \frac{I_{c}^{2} I_{v}^{2}}{(1+2 \mu)^{3 / 2}}\left(\frac{E_{e x}-E_{T}}{E_{g}}\right)^{2},
$$

where $W_{0}=4.14 \times 10^{16} \mathrm{~s}^{-1}, \varepsilon_{0}$ is the free-space permittivity, $\varepsilon_{\infty}$ is the high-frequency permittivity, $m_{c}^{*}$ is the electron effective mass, $\mu=m_{c}^{*} / m_{h}^{*}, m_{h}^{*}$ is the hole effective mass, $I_{c}$ and $I_{v}$ are the cell-periodic overlap integrals, $\mathrm{E}_{\mathrm{T}}$ is the threshold energy and $E_{g}$ is the band- gap energy ${ }^{17}$. In a parabolic system, $\mathrm{E}_{\mathrm{T}}$ is given by $E_{T}=E_{g}(1+2 \mu) /(1$ $+\mu)^{1}$. Using $m_{h}^{*}$ of the $\mathrm{LH}$ valence band in InSb, we estimate $\mathrm{E}_{\mathrm{T}}=$ $0.25 \mathrm{eV}$ at $293 \mathrm{~K}$. When we apply this formula to hot electrons with $\mathrm{E}_{\mathrm{ex}}=0.5 \mathrm{eV}$ in InSb, we get $W_{I M P}=1.1 \times 10^{12} \mathrm{~s}^{-1}$ even for $I_{c}^{2}=I_{v}^{2}=1$, establishing an upper bound. Given that Eq. (1) neglects any screening effects ${ }^{1}$, this theory underestimates the rate significantly. We presume that the discrepancy comes from the parabolic-band approximation in Eq. (1). As seen in Fig. 2(d), the dispersion of the conduction band and LH band is not parabolic, but can be well approximated by a linear dispersion in the energy range from -0.5 to $1.0 \mathrm{eV}$, except for the gap region. Dispersions along $\Gamma-\mathrm{X}$ and $\Gamma-\mathrm{K}$ directions show the same characteristic ${ }^{18}$. Therefore, momentum and energy conservations can be satisfied for the four quantum states involved in IMP processes, without any limitations to the initial impacting electronic state except $\mathrm{E}_{\mathrm{ex}}$ $>\mathrm{E}_{\mathrm{T}}$. This would make the rate of IMP significantly larger when compared to the parabolic case, as we observe.

In conclusion, we have demonstrated the state-selective features in IMP processes for the first time, and made it possible to compare experimental results and theoretical modeling of IMP rates quantitatively. Our study, based on direct measurements of energy and momentum-resolved electron distributions, provides far deeper insight into the IMP processes in semiconductors than previous studies, and gives a sound basis for investigating the current topic of ultrafast interaction of intense $\mathrm{THz}$ waves with matter ${ }^{9-11}$.

\section{Methods}

Non-doped InSb (100) wafers were cleaved under ultrahigh vacuum conditions $(<5$ $\times 10^{-11}$ Torr). Surface structures were characterized in situ by a scanning tunneling microscope prior to photoemission measurements ${ }^{22}$. The (110) surfaces displayed well-ordered $(1 \times 1)$-structure with linear In and Sb rows, and a surface-defect concentration less than $0.5 \%$. We used two different femtosecond laser systems. A mode-locked Ti:sapphire laser, operated at a $76 \mathrm{MHz}$ repetition rate, was used to generate fs-laser pulses between $820-730 \mathrm{~nm}$. The fundamental and its third har- 

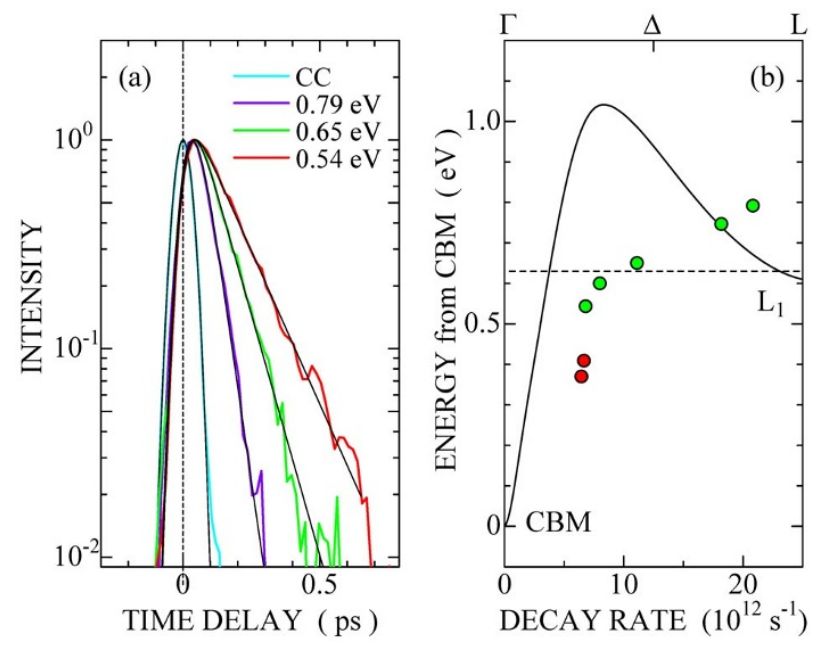

Figure $3 \mid$ Decay times of hot electrons and relation to excess energy. (a) Temporal changes in the photoemission intensities from hot electrons injected at $\mathrm{E}_{\mathrm{ex}}=0.79,0.65$ and $0.54 \mathrm{eV}$ (violet, green, and red lines, respectively) plotted on a semi-logarithmic scale. The blue curve, labelled CC, shows the cross correlation trace between pump and probe pulses, which defines the zero time delay. Measurements were made at $293 \mathrm{~K}$. The overlap between pump and probe pulses can be neglected at $\Delta \mathrm{t}>150 \mathrm{fs}$. Decay times can be determined almost uniquely from the plot, although the analysis for a whole temporal domain including rise time needs more sophisticated methods using the optical Bloch equation (see supplementary information). The thin black solid lines show results of analysis using the optical Bloch equation. (b) The relationship between transition rate, defined by the inverse of the decay time, of hot electrons and excess energy. Green and red dots are for the hot electrons injected from light-hole and split-off valence bands, respectively. The solid curve shows the dispersion of the conduction band along the $\Gamma$-L direction (after Ref. 18). The theoretical position of $\mathrm{L}_{1}$ is $0.61 \mathrm{eV}$ above the CBM, while experimentally determined value is $0.63 \mathrm{eV}$ above the CBM as shown by the broken line.

monic, generated using beta-barium borate crystals, were used as pump and probe pulses. Temporal widths of the pump and the probe pulses were $80 \mathrm{fs}$ and $100 \mathrm{fs}$ at the sample position in the UHV chamber, giving a cross-correlation trace of $125 \mathrm{fs}$. To generate shorter pulse widths with wider tunability, we also used a laser system consisting of a Ti-sapphire laser oscillator, a regeneratively amplified Ti:Sapphire laser, and a tunable optical parametric amplifier (OPA) operated at 250-kHz repetition rate. The OPA generated 50 -fs laser pulses at photon energies tunable from 1.2 to $2.4 \mathrm{eV}$. A part of the amplified fundamental output at $790 \mathrm{~nm}$ was used to generate the third harmonic with a temporal width of $65 \mathrm{fs}$. Pump and probe pulses, with a preset time delay $(\Delta t)$, were aligned co-axially and focused on the sample surfaces at $45^{\circ}$ to normal.

The ionization energy of $\operatorname{InSb}(110)$ is $4.77 \mathrm{eV}^{23}$. Therefore, the third harmonic of $790-\mathrm{nm}$ fundamental beam $(4.71 \mathrm{eV})$ can probe the states populated in the conduction band, but cannot ionize those in the valence band. This discrimination is very important to capture accurately the photoinjected hot electrons in the conduction band, with completely suppressing intense signals due to direct photoionization from the valence band.

For photoemission spectroscopy, a hemispherical electron analyzer, equipped with an angle-resolved lens mode and a two-dimensional image-type detector served as the electron spectrometer. The entrance slit of the analyzer was placed on the optical plane defined by incoming and reflected light. Two-dimensional images of photoelectrons were recorded as functions of energy and of emission angle $\theta$ along the [001] crystallographic direction. Here, $\theta$ corresponds to a surface-parallel wavevector $\mathrm{k}_{/ /}$ along the $\bar{\Gamma}-\bar{Y}$ direction of the surface Brillouin zone (SBZ), on which all states along the $\Gamma$-X and $\Gamma$-L lines, in the bulk Brillouin zone, are projected with finite values of $\mathrm{k}_{/ /}$. The instrumental energy resolution with fs-probe light was $45 \mathrm{meV}$, while angle resolution was limited in the range of $\pm 1^{\circ}$.

1. Ridley, B. K. Quantum Processes in Semiconductors. (Oxford University Press, Oxford, 1999).

2. Robbins, D. J. Aspects of the Theory of Impact Ionization in Semiconductors (1). phys. stat. sol. (b) 97, 9-50 (1980).
3. Ferry, D. K. Semiconductor Transport. (Taylor \& Francis, London, 2000).

4. Würfel, P. Solar energy conversion with hot electrons from impact ionization. Solar Energy Materials and Solar Cells 46, 43-52 (1997).

5. R, D. Schaller, R. D. \& Klimov, V. I. High Efficiency Carrier Multiplication in PbSe Nanocrystals: Implications for Solar Energy Conversion. Phys. Rev. Lett. 92, 186601-1-4 (2004).

6. Tielrooij, K. J. et al. Photoexcitation cascade and multiple hot-carrier generation in graphene. Nature Physics 9, 248-252 (2013).

7. Dick, C. L. \& Ancker-Johnson, B. Nonequilibrium Carrier Phenomena in n Typ-e InSb. Phys. Rev. B 5, 526-544 (1972).

8. Eklund, E. A. et al. Direct Determination of Impact-Ionization Rates near Threshold in Semiconductors Using Soft-X-Ray Photoemission. Phys. Rev. Lett. 68, 831-834 (1992)

9. Cartier, E., Fischetti, M. V., Eklund, E. A. \& McFeely, F. R. Impact ionization in silicon. Appl. Phys. Lett. 62, 3339-3341 (1993).

10. Wen, H., Wiczer, M. \& Lindenberg, A. M. Ultrafast electron cascades in semiconductors driven by intense femtosecond terahertz pulses. Phys. Rev. B 78, 125203-1-6 (2008).

11. Hoffmann, M. C. et al. Impact ionization in InSb probed by terahertz pumpterahertz probe spectroscopy. Phys. Rev. B 79, 161201-1-4 (2009).

12. Ho, I-Chen \& Zhanga, X.-C. Driving intervalley scattering and impact ionization in InAs with intense terahertz pulses. Appl. Phys. Lett. 98, 241908-1-3 (2011).

13. Sano, N. \& Yoshii, A. Impact-ionization model consistent with the band structure of semiconductors. J. Appl. Phys. 77, 2020-2025 (1995).

14. Picozzi, S., Asahi, R., Geller, C. B. \& Freeman, A. J. Accurate First-Principles Detailed-Balance Determination of Auger Recombination and Impact Ionization Rates in Semiconductors. Phys. Rev. Lett. 89, 197601-1-4 (2002).

15. Kotani, T. \& van Schilfgaarde, M. Impact ionization rates for Si, GaAs, InAs, ZnS, and GaN in the GW approximation. Phys. Rev. B 81, 125201-1-5 (2010)

16. Asmontaš, S., Raguotis, R. \& Bumeliené, S. Monte Carlo calculations of the electron impact ionization in n-type InSb crystal. Semicond. Sci. Technol. 28, 025019-1-3 (2013).

17. Ridley, B. K. Consequences of Wannier-Stark quantization on the impact ionization rate in insulators and large-bandgap semiconductors. J. Phys.: Condens. Matter 10, L607-L613 (1998).

18. Malone, B. D. \& Cohen, M. L. Quasiparticle semiconductor band structures including spin-orbit interactions. J. Phys.: Condens. Matter 25, 105503-1-13 (2013).

19. Brand, S. \& Abram, R. A. Calculations of overlap integrals for Auger processes involving direct band gap semiconductors. J. Phys. C: Solid State Phys. 17, L201-L206 (1984).

20. Burt, M. G., Brand, S., Smith, C. \& Abram, R. A. Overlap integrals for Auger recombination in direct bandgap semiconductors: calculations for conduction and heavy-hole bands in GaAs and InP. J. Phys. C: Solid State Phys. 17, 6385-6401 (1984).

21. Grober, R. D., Drew, H. D., Burdge, G. L. \& Bennett, B. S. Direct measurement of the recombination rates in bulk InSb by time-resolved photoluminescence. J. Appl. Phys. 71, 5140-5145 (1992).

22. Kanasaki, J., Inami, E. \& Tanimura, K. Fermi-level dependent morphology in photoinduced structural instability on (110) surfaces of III-V semiconductors. Surf. Sci. 601, 2367-2372 (2007).

23. Gobeli, G. W. \& Allen, F. G. Photoelectric Properties of Cleaved GaAs, GaSb, InAs, and InSb Surfaces; Comparison with Si and Ge. Phys Rev. 137, A245-A254 (1965).

\section{Acknowledgments}

This work was supported by a Specially Promoted Research (24000006) of Grant-in-Aid for Scientific Research from the JSPS, Japan.

\section{Author contributions}

H.T. and J.K. conducted the experiments, analyzed data and wrote the manuscript. K.T. discussed the results and interpretation, and the manuscript.

\section{Additional information}

Supplementary information accompanies this paper at http://www.nature.com/ scientificreports

Competing financial interests: The authors declare no competing financial interests.

How to cite this article: Tanimura, H., Kanasaki, J. \& Tanimura, K. State-resolved ultrafast dynamics of impact ionization in InSb. Sci. Rep. 4, 6849; DOI:10.1038/srep06849 (2014).

This work is licensed under a Creative Commons Attribution-NonCommercialNoDerivs 4.0 International License. The images or other third party material in this article are included in the article's Creative Commons license, unless indicated otherwise in the credit line; if the material is not included under the Creative Commons license, users will need to obtain permission from the license holder in order to reproduce the material. To view a copy of this license, visit http:// creativecommons.org/licenses/by-nc-nd/4.0/ 